\title{
FACTIBILIDAD TÉCNICA DE LA UTILIZACIÓN DE COBERTURA VEGETAL EN PAPAYA (Carica papaya L.) MEDIANTE LA APLICACIÓN LOCALIZADA DE HERBICIDAS 1
}

\author{
Antonio Bogantes ${ }^{2}$ Eric Mora ${ }^{3}$
}

\begin{abstract}
RESUMEN
Factibilidad técnica de la utilización de cobertura vegetal en papaya (Carica papaya $\mathrm{L}$.) mediante la aplicación localizada de herbicidas. Se estudió la factibilidad de utilizar cobertura vegetal en papaya (Carica papaya L.) bajo un sistema de siembra sobre lomillos. El objetivo fue establecer un área libre de malezas sobre el lomillo para permitir la colocación del fertilizante cerca del sistema radical de la papaya. Se evaluó el control de malezas de cuatro herbicidas (gifosato, oxifluorfen, pendimetalina y acetaclor) sobre especies de hoja ancha, poáceas y ciperáceas, así como el posible daño de los herbicidas al cultivo y su efecto sobre el crecimiento y rendimiento de las plantas de papaya. El herbicida glifosato fue eficiente en el control de poáceas pero no contra hojas anchas. Se obtuvo muy buen control general de malezas hasta los 90 después de la aplicación con los tratamientos preemergentes oxifluorfen+pendimetalina y acetaclor. Los dos primeros herbicidas afectaron levemente las plantas de papaya cuando se aplicaron a los 10 días después de la siembra. El acetaclor no causó daño en las plantas de papaya en ninguna de las aplicaciones. No hubo diferencias entre los tratamientos con respecto a las variables de crecimiento y rendimiento del cultivo.
\end{abstract}

Palabras claves: Carica papaya, lomillos, herbicidas, sitio de fertilización.

\begin{abstract}
Technical feasibility of the use of groundcover in papaya (Carica papaya L.) by means of the localized application of herbicides. An essay was conducted to evaluate the feasibility of implementing the use of natural groundcover in papaya (Carica papaya L.) under a ridge planting system. The objective was to establish a weed-free zone on the ridge area to allow the placement of fertilizer close to the root systems, thus avoiding competition between the roots of the papaya and those of the groundcover. Four herbicides were used (acetachlor, pendimethaline, oxyfluorfen and glyphosate) to asses their control of cyperous, broadleaf and grass weeds. Possible damage to papaya plants due to the effect of the herbicides was also monitored as well and their response in terms of growth and yield. Glyphosate was efficient for gramineous weed control but not against broadleaves. Very good control for all weed types was obtained during the 90 day post-transplant period with the oxyfluorfen + pendimethaline and acetachlor treatments. The first two herbicides affected papaya plants slightly when they were applied 10 days after transplant. Acetachlor didn't cause any damage to the papaya. There were no differences among the treatments in regard to growth and yield.
\end{abstract}

Key words: Carica papaya, ridge planting, herbicides, fertilization place.

\section{INTRODUCCIÓN}

Existe una fuerte preocupación a nivel mundial por aspectos de sostenibilidad ambiental durante el proceso productivo agrícola. Un claro ejemplo de esta situación se puede observar con la inclusión de regulaciones pa- ra la protección del suelo a través de diversas normativas, tales como el EUREPGAP (EUREP 2004).

La erosión del suelo es una de las amenazas más fuertes a la producción sostenible desde el punto de vista ambiental. En nuestro país, cerca del $42 \%$ del área

\footnotetext{
1 Recibido para publicación el 24 de junio del 2004.

2 Estación Experimental Los Diamantes, INTA-MAG. Costa Rica.

3 Estación Experimental Fabio Baudrit, Universidad de Costa Rica. Costa Rica. emoran@racsa.co.cr
} 
agrícola se encuentra amenazada por los procesos de erosión hídrica (Dercksen 1991).

El sistema tradicional de siembra de papaya en Costa Rica consiste en el establecimiento de la plantación sobre suelo totalmente descubierto y la fertilización superficial en forma circular sobre el perímetro de la proyección de la copa. Este sistema se utiliza debido a que la papaya presenta un sistema radical muy superficial (Masr 1993) que no tolera la competencia de malezas, potenciales protectoras del suelo contra la erosión. Por este motivo, la recomendación tradicional de control de malezas se basa en la aplicación de los productos paraquat y glifosato (Guzmán 1998) en forma total (bandas y entrecalles) pero evitando el cultivo.

El sistema tradicional tampoco contempla el uso de lomillos o domos, a pesar de la alta pluviosidad que impera en las zonas típicamente productoras en Costa Rica (entre 2.500 y $4.500 \mathrm{~mm}$ anuales). Este factor es de mucha importancia durante la etapa de establecimiento del cultivo, debido a la alta susceptibilidad de esta especie al patógeno de suelo Phytophtora sp. Teóricamente, la colocación del fertilizante de forma más localizada en el lomillo también permitiría el mantenimiento de la entrecalle con una cobertura vegetativa espontánea, al disminuir las posibilidades de competencia entre las raíces del cultivo y la cobertura vegetal. La utilización de coberturas vegetales en las entrecalles ha mostrado viabilidad en otros cultivos como la pimienta (Bogantes y Delgado 1998) y el palmito (Bogantes 2004), al no afectar negativamente su potencial de rendimiento.

El objetivo de este estudio fue el de analizar estrategias de control de malezas y métodos de fertilización con potencial para prevenir la erosión de suelo sin afectar la eficiencia del uso del fertilizante.

\section{MATERIALES Y MÉTODOS}

\section{Descripción del área de investigación}

El estudio se realizó entre marzo del 2003 y marzo del 2004, en la finca de un productor en la Rita de Guápiles, Pococí, en el Caribe de Costa Rica a una altitud de $249 \mathrm{msnm}$. Los datos climáticos durante el período de estudio se muestran en el Cuadro 1. La precipitación promedio anual es de $4.500 \mathrm{~mm}$ para dicha región.

\section{Preparación del terreno y siembra}

Se realizó una pasada de arado y otra de rastra y se hicieron lomillos espaciados a 2,5 m entre cada uno. Se
Cuadro 1. Promedio de datos climáticos en el período del estudio. Guápiles, Costa Rica. Marzo 2003 - febrero 2004.

\begin{tabular}{lrcc}
\hline \multicolumn{1}{c}{ Mes } & $\begin{array}{c}\text { Precipitación } \\
(\mathbf{m m})\end{array}$ & $\begin{array}{c}\text { Temperatura } \\
\text { media }\left({ }^{\circ} \mathbf{C}\right)\end{array}$ & $\begin{array}{c}\text { Humedad } \\
\text { relativa }(\%)\end{array}$ \\
\hline Marzo 2003 & 151,8 & 25,1 & 84 \\
Abril & 247,3 & 25,2 & 85 \\
Mayo & 277,5 & 25,2 & 89 \\
Junio & 355 & 25,1 & 84 \\
Julio & 522 & 24,9 & 89 \\
Agosto & 390,8 & 25,1 & 87 \\
Setiembre & 155 & 24,9 & 85 \\
Octubre & 197 & 25 & 87 \\
Noviembre & 250,4 & 24,5 & 88 \\
Diciembre & 510,5 & 25,5 & 90 \\
Enero 2004 & 199,5 & 23,5 & 85 \\
Febrero & 344,5 & 23,9 & 84 \\
\hline
\end{tabular}

Fuente: CORBANA, Estación La Rita de Guápiles, Costa Rica.

transplantaron plantas del híbrido "Pococî", de dos semanas de edad. Para la evaluación preliminar de los herbicidas, se sembraron plantas a $0,50 \mathrm{~m}$ entre sí, y en los tratamientos definitivos se sembró a 2,5 m entre plantas.

\section{Tratamientos y diseño experimental}

Se hizo una prueba preliminar con plantas de 10 días de sembradas, para seleccionar los tratamientos herbicidas (Cuadro 2).

Cuadro 2. Herbicidas preemergentes utilizados durante etapa preliminar para determinar productos en ensayo definitivo. Guápiles, Costa Rica. 2004.

\begin{tabular}{lr}
\hline \multicolumn{1}{c}{ Herbicidas } & $\begin{array}{c}\text { Dosis } \\
\text { (producto comercial) }\end{array}$ \\
\hline Acetaclor & $2,5 \mathrm{~kg}$ i.a. / ha \\
Atrazina & $1,5 \mathrm{~kg} \mathrm{i.a.} / \mathrm{ha}$ \\
Pendimetalina & $1,0 \mathrm{~kg} \mathrm{i.a.} \mathrm{/} \mathrm{ha}$ \\
Oxifluorfen & $1,0 \mathrm{~kg}$ i.a. / ha \\
Atrazina + pendimetalina & $1,5 \mathrm{~kg}$ i.a. / ha $+1.0 \mathrm{~kg}$ i.a. / ha \\
Oxifluorfen + pendimetalina & $1,0 \mathrm{~kg}$ i.a. / ha $+1.0 \mathrm{~kg}$ i.a. / ha \\
\multicolumn{2}{l}{ Sin control de malezas (testigo) } \\
\hline
\end{tabular}

Después de seleccionar los herbicidas a utilizar se establecieron los tratamientos definitivos (Cuadro 3).

Para los tratamientos 2,3 y 4 de la prueba definitiva, la fertilización se realizó con espeque sobre 
Cuadro 3. Herbicidas seleccionados durante la etapa preliminar para su utilización en el ensayo definitivo. Guápiles, Costa Rica. 2004.

\begin{tabular}{cll}
\hline $\begin{array}{c}\text { Tratamiento } \\
(\#)\end{array}$ & \multicolumn{1}{c}{$\begin{array}{c}\text { Herbicidas } \\
\text { (dosis) }\end{array}$} & $\begin{array}{c}\text { Fertilización } \\
\text { (sitio) }\end{array}$ \\
\hline 1 & Glifosato $(1 \mathrm{~kg} \mathrm{i.a./ha})$ total* & Rodaja \\
2 & Glifosato $(1 \mathrm{~kg}$ i.a./ha $)$ al lomillo & Lomillo \\
3 & Oxifluorfen+pendimetalina & \\
& $(1,0+1,0 \mathrm{~kg} \mathrm{i.a.} /$ ha $)$ & Lomillo \\
4 & Acetaclor $(2,5 \mathrm{~kg}$ i.a. $/ \mathrm{ha})$ & Lomillo \\
\hline
\end{tabular}

* Aplicación total (sobre lomillo y entrecalle) como la hace el productor.

el lomillo a ambos lados de la gotera que forma la hoja. En el tratamiento 1 la fertilización se realizó en rodaja alrededor de la planta, tal y como la realiza tradicionalmente el productor.

El plan de fertilización utilizado se basó en el manejo tradicional del productor y consistió en la aplicación de 10-30-10 a la siembra y un mes después de la siembra (mdds); cal dolomítica (tres mdds); 18-5-15-6-1.2 (tres, cuatro y cinco mdds); $15-3-31$ (seis mdds) y $\mathrm{KCl}$ (siete y ocho mdds). En total se aplicó el equivalente a $210 \mathrm{~kg}$ de $\mathrm{N}, 100 \mathrm{~kg}$ de $\mathrm{P}_{2} \mathrm{O}_{5}$ y $530 \mathrm{~kg}$ de $\mathrm{K}_{2} 0$. En la prueba preliminar la aplicación de los herbicidas se hizo alrededor de la planta (60 cm de diámetro). En el estudio definitivo la aplicación fue total en el tratamiento comercial o dirigida a todo el lomillo en los tratamientos con preemergente. Se usó una bomba de mochila marca Field King de 14 litros con una boquilla TJ 8002.

Los tratamientos con herbicida glifosato (1 y 2 ) se aplicaron a los 30 y 90 días después de la siembra (dds), mientras que los tratamientos ( 3 y 4 ) se aplicaron a los 30 y 120 dds.

En el estudio preliminar de los herbicidas, los siete tratamientos se distribuyeron en ocho repeticiones totalmente al azar (una planta por repetición); mientras que, en el estudio definitivo, los cuatro tratamientos se distribuyeron en bloques completos al azar con cuatro repeticiones. La parcela consistió en dos hileras de plantas de 20 metros cada una.

\section{Las variables evaluadas fueron:}

1) Estudio preliminar: porcentaje de cobertura de hojas anchas, poáceas, ciperáceas y total en el área aplicada a los 25 días después de la aplicación (dda), daño causado por los herbicidas a las plantas de papaya a los 12 y 25 dda. Para evaluar este daño se utilizó una escala visual de 1 a 5 ; en donde: $1=\sin$ daño, $2=$ leve (1$25 \%), 3=$ moderado $(26-50 \%)$, $4=$ severo $(51-75 \%), 5=$ muy severo (más de 75\%). Además se midió la altura (cm) de plantas de papaya al inicio y a los 45 dda.

2) Estudio definitivo: especies presentes, porcentaje de cobertura de hojas anchas, poáceas, ciperáceas y total en el área aplicada a los 60 días después de la aplicación (dda); así como daño visual causado por los herbicidas a las plantas de papaya en las dos aplicaciones.

La estimación de la cobertura de malezas se realizó de forma visual y se usó un marco de $0,25 \mathrm{~m}^{2}$ el cual se lanzó tres veces por parcela útil en la prueba preliminar y dos en la prueba definitiva.

También se evaluaron las siguientes variables en la planta: altura y grosor del tallo $(\mathrm{cm})$ a los $3 \mathrm{mds}$; número total de frutos en la primera cosecha (tres plantas/parcela útil) $8 \mathrm{mds}$, peso de frutas (g) y brix a los 11 mds (tres frutas/parcela útil). La altura se midió desde la base hasta el crecimiento terminal o ápice. El grosor del tallo se midió a un metro del suelo.

El análisis estadístico de las variables se hizo con el programa SAS y para la separación de medias se utilizó la prueba de D.M.S. al 5\%.

\section{RESULTADOS}

\section{Estudio preliminar}

No hubo diferencias entre herbicidas preemergentes con respecto al control de malezas poáceas, hojas anchas, ciperáceas o total de éstas hasta los 25 dda. En esta etapa de preselección de los tratamientos, se puede concluir de manera general, que los preemergentes evaluados tuvieron un buen control de las malezas con respecto al testigo, aún después de la evaluación a los 25 dda (Cuadro 4).

El preemergente atrazina causó daño severo a las plantas de papaya, el cual consistió en una quema de hasta el $75 \%$ de la planta, tanto a los 12 dda como a los 25 dda (Cuadro 5). En algunos casos estas plantas llegaron a morir, por lo cual dicho tratamiento no se consideró en el análisis de varianza para la variable altura de planta. La atrazina es un herbicida contra poáceas y controla algunas hojas anchas, se absorbe del suelo vía radical y es traslocado por el apoplasto (Vencill 2002). La selectividad de este herbicida a cultivos como maíz, caña de azúcar y sorgo estriba en la habilidad de degradar bioquímicamente el herbicida a metabolitos no 
Cuadro 4. Porcentaje de poáceas, hojas anchas y ciperáceas en papaya, 25 dda. Estudio preliminar, Guápiles, Costa Rica. 2004.

\begin{tabular}{lrccc}
\hline $\begin{array}{c}\text { Herbicidas } \\
\text { (tratamiento) }\end{array}$ & $\begin{array}{c}\text { Poáceas } \\
\mathbf{p} \leq \mathbf{0 , 2 1}\end{array}$ & $\begin{array}{c}\text { Hoja ancha } \\
\mathbf{p} \mathbf{\leq 0 , 0 0 0 1}\end{array}$ & $\begin{array}{c}\text { Ciperáceas } \\
\mathbf{p} \leq \mathbf{0 , 4 3}\end{array}$ & $\begin{array}{c}\text { Total } \\
\mathbf{0 , 0 0 0 1}\end{array}$ \\
\hline 1. Acetaclor & 2,25 & $0,50 \mathrm{a}$ & 0,00 & $2,75 \mathrm{a}$ \\
2. Atrazina & 1,5 & $0,00 \mathrm{a}$ & 0,00 & $1,50 \mathrm{a}$ \\
3. Pendimetalina & 4,12 & $0,00 \mathrm{a}$ & 0,00 & $4,12 \mathrm{a}$ \\
4. Oxifluorfen & 2,57 & $0,28 \mathrm{a}$ & 0,00 & $2,85 \mathrm{a}$ \\
5. Atrazina + pendimetalina & 3,00 & $0,25 \mathrm{a}$ & 0,00 & $3,25 \mathrm{a}$ \\
6. Oxifluorfen+pendimetalina & 5,44 & $0,11 \mathrm{a}$ & 0,00 & $5,55 \mathrm{a}$ \\
7. Testigo libre crecimiento & & & & \\
$\quad$ de malezas (testigo) & 8,37 & $6,87 \mathrm{~b}$ & 0,12 & $15,38 \mathrm{~b}$ \\
\hline
\end{tabular}

Cuadro 5. Porcentaje de daño en plantas de papaya por diferentes herbicidas 12 y 25 después de aplicación. Estudio preliminar, Guápiles, Costa Rica. 2004.

\begin{tabular}{lcc}
\hline $\begin{array}{c}\text { Herbicidas } \\
\text { tratamiento) }\end{array}$ & $\begin{array}{c}\text { Daño } \\
\mathbf{1 2} \mathbf{~ d d a ~ ( \% )} \\
\mathbf{p = 0 , 0 3}\end{array}$ & $\begin{array}{c}\text { Daño } \\
\mathbf{2 5} \mathbf{~ d d a ~}(\%) \\
\mathbf{p}<\mathbf{0 , 0 0 0 1}\end{array}$ \\
\hline 1. Acetaclor & $0,62 \mathrm{c}$ & $1,25 \mathrm{c}$ \\
2. Atrazina & $14,37 \mathrm{abc}$ & $78,13 \mathrm{a}$ \\
3. Pendimetalina & $17,50 \mathrm{ab}$ & $48,75 \mathrm{~b}$ \\
4. Oxifluorfen & $3,00 \mathrm{bc}$ & $0,71 \mathrm{c}$ \\
5. Atrazina + pendimetalina & $10,00 \mathrm{abc}$ & $5,00 \mathrm{c}$ \\
6. Oxifluorfen+pendimetalina & $20,00 \mathrm{a}$ & $18,33 \mathrm{c}$ \\
7. Testigo & $0 \mathrm{c}$ & $1,25 \mathrm{c}$ \\
\hline
\end{tabular}

tóxicos (Hilton 1970, Lamoureux 1970 y Montgomery 1961 citados por Jensen et al. 1977).

El herbicida pendimetalina aplicado al suelo también se absorbe por las raíces (Vencill 2002). El daño por este herbicida fue leve o moderado, causando clorosis en un 25 a $50 \%$ de la planta y necrosis en los bordes. Esta sintomatología se presentó tanto al inicio como a los 25 dda (Cuadro 5). Sin embargo, las plantas mostraron luego una recuperación, tal y como se refleja en el incremento de la altura (Cuadro 6). Los menores porcentajes de daños a los 25 dda lo mostraron las plantas en las que se usaron los preemergentes oxifluorfen, acetaclor y atrazina+ pendimetalina. No obstante, debido al severo efecto de la atrazina sobre las plantas de papaya, este preemergente se descartó en el estudio definitivo. A los 12 dda, las plantas en las que se usó la mezcla oxifluorfen+pendimetalina, presentaron dos o tres hojas hojas con epinastia y/o con necrosis en los bordes. Sin embargo, a los 25 dda estas plantas mostraron una total recuperación. Es importante mencionar que el oxifluorfen es el único herbicida preemergente recomendado para papaya (Vencill 2002).
Cuadro 6. Incremento de altura $(\mathrm{cm})$ en plantas de papaya con diferentes tratamientos herbicidas 45 dda. Estudio preliminar. Guápiles, Costa Rica. 2004.

\begin{tabular}{lc}
\hline $\begin{array}{c}\text { Herbicidas } \\
\text { (tratamiento) }\end{array}$ & $\begin{array}{c}\text { Incremento de altura } \\
(\mathbf{p = 0 , 0 2})\end{array}$ \\
\hline 1. Acetaclor & $49,56 \mathrm{a}$ \\
2. Atrazina & $*$ \\
3. Pendimetalina & $35,25 \mathrm{~b}$ \\
4. Oxifluorfen & $44,00 \mathrm{ab}$ \\
5. Atrazina + pendimetalina & $52,25 \mathrm{a}$ \\
6. Oxifluorfen + pendimetalina & $48,14 \mathrm{a}$ \\
7. Testigo & $52,62 \mathrm{a}$ \\
\hline
\end{tabular}

* No se incluyó en el análisis por muerte de plantas.

El incremento en altura de las plantas de papaya fue menor en los tratamientos con pendimetalina y oxifluorfen solos, no obstante, en el tratamiento con ambos preemergentes en mezcla, la diferencia en altura no fue significativa con respecto al testigo con agua (Cuadro 6).

Con base en estos resultados se seleccionaron los preemergentes acetaclor, pendimetalina y oxifluorfen para ser evaluados en el estudio definitivo junto con el glifosato (testigo usado por el productor).

\section{Estudio definitivo}

En el Cuadro 7 se observan los datos de la cobertura de malezas total y en grupos, según los herbicidas utilizados. Es evidente a los 60 dda la diferencia entre los tratamientos con el herbicida glifosato (1 y 2) aplicado en posemergencia con respecto a los tratamientos preemergentes (3 y 4). El glifosato controló efectivamente las malezas poáceas hasta los 60 dda (tratamientos 1 y 2), pero no así las hojas anchas. Esto incidió en 
Cuadro 7. Porcentaje de cobertura de especies poáceas, hojas anchas y ciperáceas en el lomillo según tratamientos, 60 dda. Guápiles, Costa Rica. 2004.

\begin{tabular}{lcccc}
\hline \multicolumn{1}{c}{ Tratamiento } & $\begin{array}{c}\text { Poáceas } \\
\mathbf{p} \mathbf{0 0 , 0 0 0 1}\end{array}$ & $\begin{array}{c}\text { Hoja ancha } \\
\mathbf{p} \leq \mathbf{0 , 0 0 0 1}\end{array}$ & $\begin{array}{c}\text { Ciperáceas } \\
\mathbf{p} \leq \mathbf{0 , 0 4}\end{array}$ & $\begin{array}{c}\text { Total } \\
\mathbf{p} \leq \mathbf{0 , 0 0 0 1}\end{array}$ \\
\hline $\begin{array}{l}\text { 1. Glifosato total y fertilización rodaja } \\
\text { 2. Glifosato y fertilización al lomillo }\end{array}$ & $0,87 \mathrm{~b}$ & $60,00 \mathrm{a}$ & $1,81 \mathrm{a}$ & $62,62 \mathrm{a}$ \\
$\begin{array}{l}\text { 3. Oxifluorfen+pendimetalina y } \\
\quad \text { fertilización al lomillo }\end{array}$ & $0,25 \mathrm{~b}$ & $0,18 \mathrm{c}$ & $0,00 \mathrm{~b}$ & $0,12 \mathrm{~b}$ \\
4. Acetaclor y fertilización al lomillo & $0,38 \mathrm{~b}$ & $1,94 \mathrm{c}$ & $0,06 \mathrm{~b}$ & $2,25 \mathrm{~b}$ \\
\hline
\end{tabular}

la cobertura total de malezas en ese período. El glifosato se considera como un herbicida de amplio espectro y controla muy bien especies poáceas (Fernández y Ortiz 1995).

Algunas malezas de hábito estolonífero, como $C y$ nodon dactylon e Ischaemun ciliaris, crecieron en los centros de las hileras en las que no se aplicó herbicida. Este hábito de crecimiento les permitió cubrir parte del lomillo, desplazando con ello especies de hoja ancha. Lo anterior se refleja en una menor cobertura de especies de este grupo en dicho tratamiento.

Las especies presentes en el área experimental se presentan en el Cuadro 8.

Los tratamientos preemergentes tuvieron muy buen control sobre todas las malezas durante ese período evaluado (Cuadro 7). El acetaclor combatió bien incluso al- gunas especies de hoja ancha (Cuadro 7) y no fue fitotóxico para el cultivo en ambos estudios.

Es importante notar que la segunda aplicación con este herbicida se realizó hasta 90 días después de la primera, mientras que la segunda aplicación de glifosato se realizó a los 60 días después de la primera. Lo anterior es relevante porque la planta de papaya produce sombreo después de seis meses de establecimiento, el cual limita el crecimiento de las malezas y reduce los ciclos de aplicación.

La segunda aplicación de oxifluorfen+pendimetalina a los 60, produjo leves síntomas de toxicidad en la papaya. Este daño consistió en la aparición de una leve necrosis de los brotes tiernos primarios y secundarios de algunas plantas 4 dda. Sin embargo, estos efectos se redujeron a una leve necrosis en la tercera hoja $10 \mathrm{dda}$, y desaparecieron a los 15 dda. Dichos síntomas coinci-

Cuadro 8. Lista y frecuencia de especies de malezas en el lomillo, según tratamiento, 60 dda. Guápiles, Costa Rica. 2004.

\begin{tabular}{|c|c|}
\hline Tratamiento & Especies presentes \\
\hline 1 & $\begin{array}{l}\text { Ageratum conyzoides }(13 *), \text { Casia tora }(13), \text { Cynodon dactylon }(63) \text { Cyperus sp (63), } \\
\text { Digitaria sanguinalis }(25), \text { Ischaemun ciliaris }(13), \text { Lindernia crustacea }(38), \text { Lindernia } \\
\text { diffusa }(50), \text { Mecardonia procumbens }(13), \text { Melantera nivea }(25), \text { Mimosa pudica (50), } \\
\text { Phylantus niruri (75), Sida sp (13), Spermacoce sp (100). }\end{array}$ \\
\hline 2 & $\begin{array}{l}\text { Colocasia esculenta (13), Cynodon dactylon (88), Cyperus sp (63), Digitaria sanguinalis } \\
\text { (25), Ischaemun ciliaris (13), Lindernia crustacea (13) Lindernia diffusa (75), Ludwigia suf } \\
\text { fruticosa (75), Mecardonia procumbens (13), Mimosa pudica (25), Phylantus niruri (50), } \\
\text { Sida sp (13), Spermacoce sp (88). }\end{array}$ \\
\hline 3 & Colocasia esculenta (13), Cynodon dactylon (50), Phylantus niruri (13). \\
\hline 4 & $\begin{array}{l}\text { Cyperus sp (13), Cynodon dactylon (38), Emilia fosbergui (25), Mimosa pudica (38), } \\
\text { Phylantus niruri (75), Spermacoce sp (63). }\end{array}$ \\
\hline
\end{tabular}

* Frecuencia \% = número de apariciones/ocho estimaciones (dos por cada repetición). 
den con los descritos para el oxifluorfen en otros cultivos (Vencill 2002).

No hubo efecto de los tratamientos sobre la altura y el grosor de las plantas a los tres meses de la siembra (Cuadro 9) ni sobre el número de frutas, peso y brix de las mismas (Cuadro 10). Sí se presentaron diferencias significativas en cuanto al manejo de la cobertura del lomillo sobre los grados brix entre el glifosato y los herbicidas preemergentes. Una posible explicación a este fenómeno podría ser que se alterara la relación fuente-sumidero, en vista de que se ha comprobado que dicha relación puede afectar los grados brix de la fruta de papaya (Zhou et al. 2000). Para futuros trabajos con herbicidas preemergentes debe explorarse esta posibilidad, específicamente, debería investigarse un posible efecto de dichos herbicidas sobre la duración del área foliar del cultivo.

Cuadro 9. Altura y grosor de plantas de papaya, tres meses después de la siembra según tratamiento. Guápiles, Costa Rica. 2004

\begin{tabular}{|c|c|c|}
\hline Tratamiento & $\begin{array}{c}\begin{array}{c}\text { Altura } \\
(\mathrm{cm})\end{array} \\
\mathbf{p = 0 , 6 4} \text { n.s. }\end{array}$ & $\begin{array}{c}\text { Grosor } \\
(\mathrm{cm}) \\
\mathrm{p}=\mathbf{0 , 4 2} \text { n.s. }\end{array}$ \\
\hline 1. Glifosato total y fertilización rodaja & 122 & 5,2 \\
\hline 2. Glifosato y fertilización al lomillo & 121 & 5,4 \\
\hline $\begin{array}{l}\text { 3. Oxifluorfen+pendimetalina y } \\
\text { fertilización al lomillo } \\
\text { 4. Acetaclor y fertilización al lomillo }\end{array}$ & $\begin{array}{l}115 \\
122\end{array}$ & $\begin{array}{l}4,9 \\
5,4\end{array}$ \\
\hline
\end{tabular}

Cuadro 10. Número, peso y brix de frutas de papaya según tratamiento. Guápiles, Costa Rica. 2004.

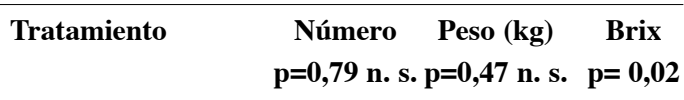

1. Glifosato total y fertilización rodaja

95

1,08

$11,07 \mathrm{ab}$

2. Glifosato y fertilización al lomillo

90

1,05

11,38 a

3. Oxifluorfen+pendimetalina y fertilización al lomillo

4. Acetaclor y fertilización al lomillo

$91 \quad 1,14 \quad 10,69 \mathrm{~b}$

\section{CONCLUSIONES}

El herbicida glifosato fue eficiente en el control de poáceas pero no contra hojas anchas. Se obtuvo buen control general de malezas hasta los 90 dda con los tratamientos preemergentes oxifluorfen+pendimetalina $\mathrm{y}$ acetaclor, Los dos primeros herbicidas afectaron levemente las plantas de papaya cuando se aplicaron a los 10 días después de la siembra (dds), no obstante éstas se recuperaron. La aplicación de estos productos a los 30 dds no causó daño a las plantas de papaya. El acetaclor no causó daño en las plantas de papaya en ninguna de las aplicaciones.

No hubo diferencias entre los tratamientos con respecto a las variables de crecimiento y rendimiento del cultivo, de lo cual se deduce la posibilidad de evitar aplicaciones totales de herbicidas y fertilización en rodaja e implementar el control de malezas con alguno de los herbicidas evaluados así como la fertilización con espeque en el lomillo (Figura 1).

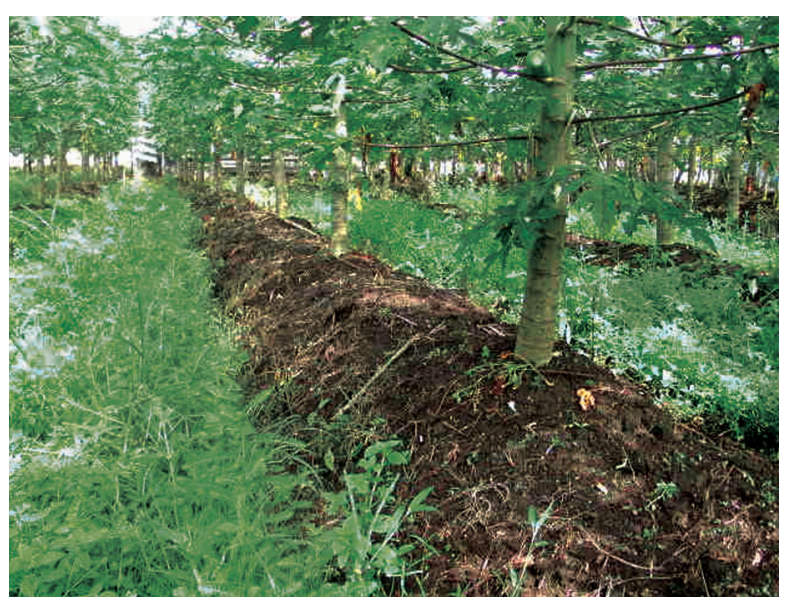

Figura 1. Tratamiento con herbicida preemergente sobre el lomillo y cobertura en la entrecalle. Se observa la factibilidad técnica de su uso a nivel comercial.

Estos resultados sugieren que es posible implementar un sistema de uso de cobertura vegetal sobre el suelo sin afectar el rendimiento del cultivo por competencia con dicha cobertura, lo cual podría disminuir la erosión hídrica común de los sistemas tradicionales de cultivo en esta especie. Se recomienda realizar más estudios para determinar la edad óptima de las plantas de papaya para la aplicación de los preemergentes oxifluorfen y pendimetalina, así como determinar el efecto de las malezas en la posible aparición de plagas y enfermedades, al servir como hospederos de éstos. 


\section{LITERATURA CITADA}

BOGANTES, A. 2004. Evaluación de cuatro distancias de siembra y dos estrategias de control de malezas en pejibaye sin espinas (Bactris gasipaes K.) para palmito. Tesis Magister Scientiae, Universidad de Costa Rica. San José, Costa Rica. 106 p.

BOGANTES, A.; DELGADO, G. 1998. Comparación de tres coberturas y dos métodos convencionales de combate de malezas en su efecto sobre algunos aspectos del cultivo de pimienta (Piper nigrum L.). Investigación Agrícola 7 (1-2):67-72.

DERCKSEN, P. 1991. A soil erosion mapping excercise in Costa Rica. In: Vahrson, W.G. (eds). Memoria: Taller de erosión de suelos. Escuela de Ciencias Geográficas, Universidad Nacional. p.164-170.

EUREP. 2004. Puntos de control y criterios de cumplimiento. frutas y vegetales. versión 2.0. Consultado: 11 junio del 2004. Disponible en: http://www.eurep.org/documents/
webdocs/EUREPGAP_CPCC_FP_V2-0Jan04_SP_update_25-02-04.pdf

FERNÁNDEZ, O.; ORTIZ, R.A. 1995. Evaluación de herbicidas graminicidas para el combate de gamalote (Paspalum fasciculatum $\mathrm{W}$.). en palma aceitera. Agronomía Mesoamericana (6): 15-22.

GUZMÁN D, G. Guía para el cultivo de papaya (Carica papaya L.). Serie cultivos no tradicionales. MAG. San José, Costa Rica. 74 p.

JENSEN, K.I.N.; STEPHENSON, G.R.; HUNT, L.A. 1977. Detoxification of atrazine in three gramineae subfamilies. Weed Science 25-3: 212-220.

MASR, M. 1993. Rooting pattern and distribution of absorbing roots of papaya (Carica papaya L.) var. Eksotica. MARDI Research Journal 21(2):99-104.

VENCILL, W. 2002. Herbicide Handbook. Eighth Edition. Weed Science Society of America. U.S.A. 482p. 